\section{SOLAR NEUTRINO PROBLEM Experiments Begin to Shed Light}

D.L. Wark of the Physics Department, Oxford University, UK, and J.S. Nico and J.F. Wilkerson of Los Alamos National Laboratory, USA, compare the latest SAGE and GALLEX results.

It is unusual in particle physics for a significant discrepancy between theory and experiment to persist for more than twenty years. Yet that is the length of time that Ray Davis and his collaborators [1] have been measuring the solar neutrino flux by determining the amount of ${ }^{37} \mathrm{Ar}$ produced during the reaction $v+{ }^{37} \mathrm{Cl} \rightarrow \mathrm{e}^{-}+{ }^{37} \mathrm{Ar}$. The average rate they detect (weighted by the cross-section for the reaction) is $2.13 \pm 0.13 \pm 0.21 \mathrm{SNU}(1 \sigma)$ where 1 Solar Neutrino Unit (SNU) $=10^{-36}$ captures/target atom/s. Models of the energy generation mechanisms and overall structure of the sun, however, predict a higher rate for this experiment. For instance, the most recent Standard Solar Model (SSM) by John Bahcall and his collaborators [2] predicts a rate of $8.0 \pm 3.0 \mathrm{SNU}(3 \sigma)$, while the group led by S. Turck-Chieze [3] calculates a rate of $6.4 \pm 1.4 \mathrm{SNU}$. The experimental side of this discrepancy has been verified by the Kamiokande large water detector operating in Japan [4], which sees only $0.48 \pm 0.05$ (stat.) \pm 0.06 (syst.) of the rate predicted for it by the SSM.

The disagreement therefore persists, but is it a disagreement that should concern particle physicists, or is it simply a problem in astrophysics? This question arises because these experiments are mainly sensitive to the higher-energy solar neutrinos which arise from the decay of ${ }^{8} \mathrm{~B}$.

\section{Explanations for the Disagreement}

The reactions which generate the ${ }^{8} \mathrm{~B}$ are extremely sensitive to the core temperature of the sun $\left(T_{C}\right)$, and only about a $10 \%$ reduction in $T_{c}$ would be necessary to explain the rate seen in the Davis experiment. While no solar model which features such a reduction in $T_{c}$ has gained general acceptance, sceptics point out that the sun may have a better idea of what physical processes are relevant than we do, and the possibility of such a reduction cannot be dismissed. Another possibility is that the electron-neutrinos are emitted in the predicted numbers, but that after their emission some fraction of them change into muon-neutrinos or tau-neutrinos, which cannot be detected by the existing experiments. This flavour mixing, which is analogous to the Cabibbo mixing between the weak and strong eigenstates of quarks, can occur if neutrinos are massive. Even a small amount of mixing could be amplified by the matter in the sun (the MSW effect [5]) to produce the observed suppression.

Supporters of this mechanism point out that it can neatly explain why the rate in the Davis experiment seems to be lower than that seen by Kamiokande, even though the Davis experiment is sensitive to lower energy neutrinos. This difference in the rates cannot be explained by a simple reduction in $T_{\mathrm{c}}$. One way to decide between these two possibilities is to observe the far more numixing was taking place. flux.

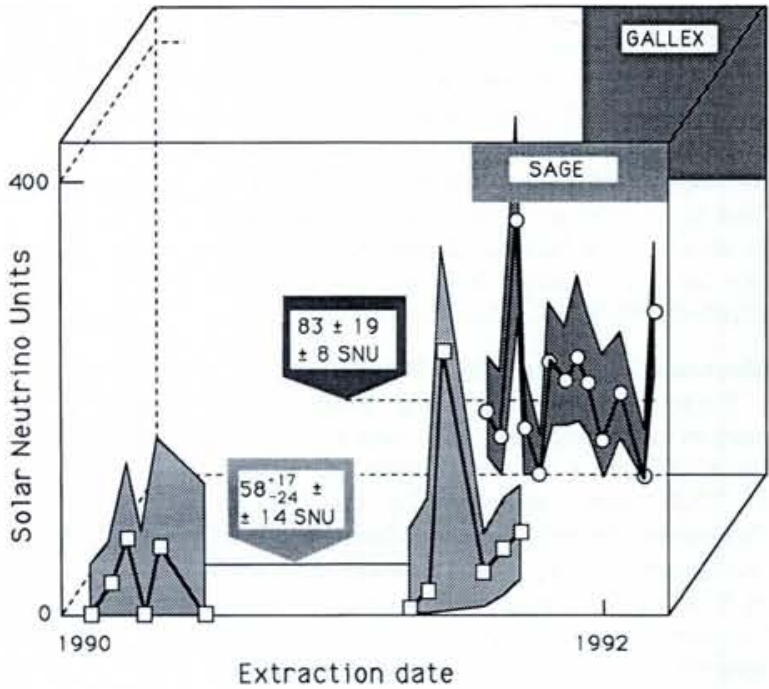

merous low-energy neutrinos coming from the first step in the proton-proton chain which powers the sun. The flux of these neutrinos is well determined by the solar luminosity, and is insensitive to the details of solar structure. A significant suppression of this flux could not be explained by any plausible solar model and would be a very strong indication that some sort of neutrino

\section{SAGE and GALLEX Results}

Two experiments (SAGE, a Soviet-American collaboration, and GALLEX, a German-French-Italian-US collaboration) are attempting to measure this flux using the reaction $v+{ }^{71} \mathrm{Ga} \rightarrow \mathrm{e}^{-}+{ }^{71} \mathrm{Ge}$, for which the SSM prediction is $132_{-17}^{+21}$ SNU [2]. Both experiments use the same basic method. First a target containing many tonnes of gallium is stored deep underground (to shield it from cosmic rays, which could produce $\mathrm{Ge}$ from the gallium by other reactions). The gallium, which contains a very small amount $(\approx 100$ 's of $\mu \mathrm{g})$ of stable germanium added as a carrier, is then exposed to solar neutrinos for several times the 11.4 day half-life of ${ }^{71} \mathrm{Ge}$. The carrier germanium and any ${ }^{71} \mathrm{Ge}$ that has been produced are then chemically extracted, concentrated, and reacted with $\mathrm{NaBH}_{4}$ to produce $\mathrm{GeH}_{4}$. The $\mathrm{GeH}_{4}$ is then mixed with enough xenon or argon to produce $=1 \mathrm{cc}$ of gas at $\approx 1 \mathrm{~atm}$. and put into a small, heavily shielded, low-background proportional counter. The carrier germanium is necessary for the counter to have sufficient gas in order to operate. These counters detect the Auger electrons or $\mathrm{x}$-rays emitted after the subsequent electron capture decay of ${ }^{71} \mathrm{Ge}$ back to ${ }^{71} \mathrm{Ga}$. Those signals which have a pulse height and rise time consistent with either L- or K-capture in ${ }^{71} \mathrm{Ge}$ are then corrected for background (real ${ }^{71} \mathrm{Ge}$ decays should show the proper half-life), and those that survive (typically less than a few counts/month) are used to determine the low-energy solar neutrino

Both experiments have now reported experimental results. The SAGE group initially reported the rate measured in 1990 [6] as $20_{-20}^{+15}$ (stat.) \pm 32 (syst.) SNU, and an upper limit of 79 SNU at the $90 \%$ confidence level.
SAGE has now added results from measurements using 57.5 tons during 1991 of [7] $85_{-24}^{+22} \pm 20$, for a combined result of $58_{-24}^{+17} \pm$ 14 SNU. The GALLEX group has 30 tonnes of gallium; their initial results indicate a rate of $83 \pm 19$ (stat.) \pm 8 (syst.) SNU [8]. Given the above values, the initial results of both experiments show reasonable agreement. These results indicate that the low-energy solar neutrino flux is also suppressed relative to calculation, although the exact amount of the suppression is not yet clear.

\section{Experimental Differences}

How do the two experiments differ? GALLEX has had the considerable statistical advantage of lower backgrounds at low pulse heights, which permits them to use the data from L-captures (SAGE has used only the K-captures and thereby doubles GALLEX's effective mass). In the $\mathrm{K}$ peak, the combination of larger target mass (for the 1991 data) and lower background gives SAGE a better signal-to-noise ratio. The SAGE experiment uses metallic gallium as the target $(30 \mathrm{t}$ for the initial results in 1990, $57.5 \mathrm{t}$ since early 1991), while GALLEX has $30 \mathrm{t}$ of gallium which they keep in the form of an aqueous solution of $\mathrm{GaCl}_{4}$. This makes the initial stages of the chemical extraction simpler for GALLEX, but the absence of hydrogen in the SAGE target makes it less sensitive to some cosmic-ray induced backgrounds. One nagging worry about both experiments is that the solar-neutrino produced ${ }^{71} \mathrm{Ge}$ may not be extracted with the same efficiency as the carrier germanium (although the different initial chemistry makes it unlikely that both experiments would be affected in the same manner). GALLEX was delayed in reporting their first results by residual ${ }^{68} \mathrm{Ge}$ (which is made by muon spallation when the gallium is on the surface), the decay of which is hard to distinguish from ${ }^{71} \mathrm{Ge}$ (SAGE experienced the same difficulty, but it was an order of magnitude smaller). GALLEX's background has now been reduced to an acceptable level.

\section{Further Work}

The lowest rate which would be consistent with nuclear fusion being the power source of the sun is $\approx 80 \mathrm{SNU}$. This rela- 
tively low predicted rate can only be obtained by stretching the errors in its calculation to their limits, and some would argue beyond. The experimental results do not unambiguously show which explanation of the solar neutrino deficit is the correct one (in fact, a result over 80 SNU could be the result of either a cool sun or neutrino mixing, so in that case other experiments would be needed). Better statistics will help, but it should be remembered that all of the results come from $\approx 100$ counts extracted from a much larger background.

Both experiments plan to calibrate their efficiencies using a powerful ${ }^{51} \mathrm{Cr}$ neutrino source. The GALLEX and SAGE experiments are constantly improving, and the next few years should bring new insight into one of particle physics' oldest and most persistent puzzles.

[1] Davis R., et al., and Lande K., 15th. Int. Conf. on Neutrino Phys. \& Astrophysics (June 1992) Granada, Spain.

[2] Bahcall J.N., ibid.

[3] Turck-Chieze S., ibid.

[4] Nakamura K., ibid., Hirata K.S., et al., Phys. Rev. Lett. 65 (1990) 1297.

[5] Mikheyev S.P. and Smirnov A.Yu., Sov. J. Nucl. Phys. 44 (1986) 938; Wolfenstein L., Phys. Rev. D 17 (1978) 2369.

[6] Abazov A.I., et al., Phys. Rev. Lett. 67 (1991) 3332.

[7] Gavrin V.N., 24th Int. Conf. on High-Energy Physics, Dallas (1992).

[8] Anselmann P., et al., Phys. Lett. B 285 (1992) 376.

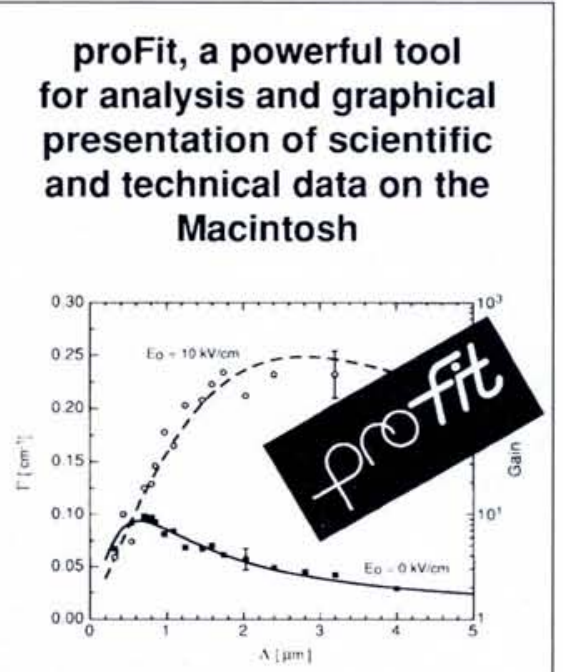

- professional graphical environment

- camera ready, high quality drawings

- highly interactive curve fitting

- built-in set of standard functions

- powerful definition syntax for custom functions

- versatile possibilities for data processing

- full support of system 7

- background operation

- reasonable price

\section{QuantumSoft}

Postfach $6613 \cdot \mathrm{CH}-8023$ Zürich

Tel. $(+41) 52 / 434174 \cdot \operatorname{Fax}(+41) 1 / 8203836$

\section{High-Mass Photon Pairs Unconfirmed - So Far}

Maurice Jacob of the Theory Division, CERN, discusses recent announcements that two of LEP's experimental collaborations have not confirmed peculiar events producing a photon pair with an invariant mass of around $60 \mathrm{GeV}$.

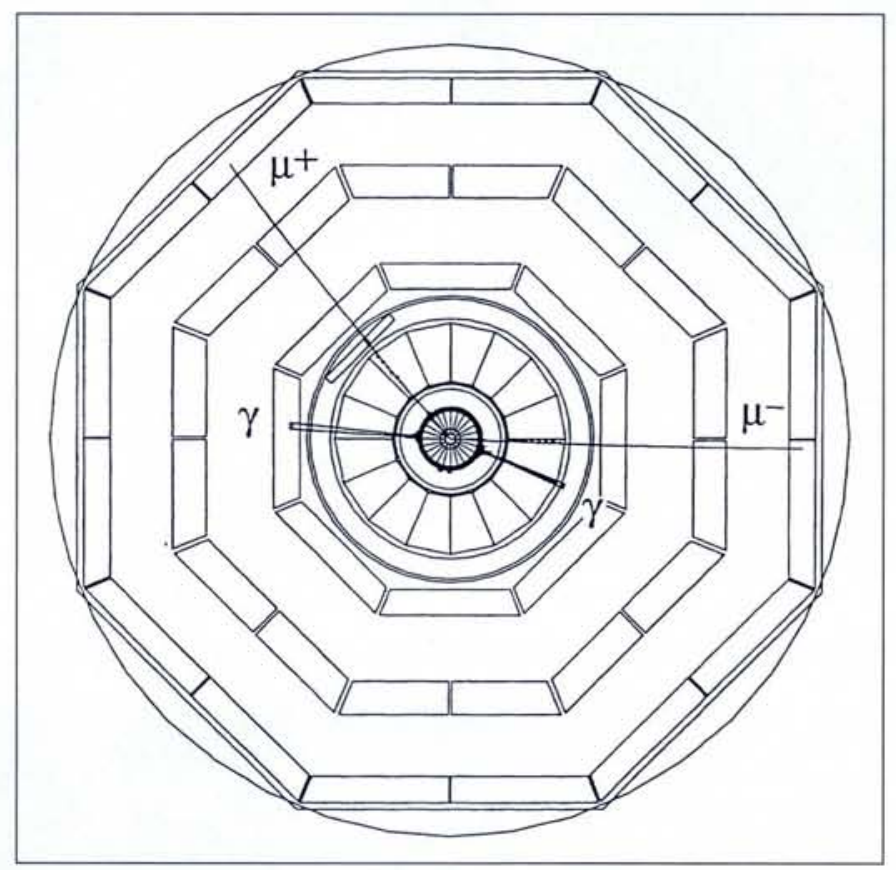

A computer visualisation of a high-mass photon event at CERN's L3 detector (the concentric details represent sub-detectors). An $e^{+} e^{-}$collision has produced a pair of photons with an invariant mass close to $60 \mathrm{GeV}$, and a pair of muons whose trajectories show that they passed into the outermost sub-detector.

The LEP collider at CERN was designed as the ideal instrument to test the Standard Model of particle physics. It has done this for three years to a precision which in some cases reaches one in a thousand. The model has held the line magnificently so physicists can look to it with pride. However, this is also frustrating since one hopes that some small failure could be the signal of hitherto unknown physics.

Each of the four LEP experiments has already recorded over one million $\mathrm{Z}$ particles so some highly improbable and unexpected features could show up. It is therefore hardly surprising that the announcement that the L3 experiment had observed four peculiar events resulted in a packed amphitheater when S.M. Ting presented them at a CERN seminar on 26 November.

The events consist of two hard photons and two leptons (two events with an electron pair and two events with a muon pair - see figure), with the invariant mass of the photon pairs clustering around $60 \mathrm{GeV}$.

While such clustering is a possible signal for the so far undiscovered Higgs particle, the observation of the two-photon effect together with only two leptons rules out this interpretation. QED processes can lead to the observed two-photon events, but the calculation of background would attach a probability of only one per million for the occurrence of all four events. Nevertheless, a QED fluctuation cannot be completely ruled out at the present time.
The events appear to be of a very peculiar type and the need for more statistics is compelling. Two events had already been seen at L3 in 1991 and two more appeared more recently. It has also become known that the DELPHI experiment had also detected an event.

The quickest way to increase statistics at LEP is to use data from all the experiments. So collaborations other than L 3 were extremely busy after the first announcement of the L3 results. What was special about the CERN seminar, besides an extensive presentation of the L3 events, was that DELPHI, ALEPH and OPAL also reported their findings.

Although DELPHI now has two-leptonic events with a two-photon invariant mass also around $60 \mathrm{GeV}$, the collaboration would not conclude at the present time from their whole set of data that there was something special. ALEPH sees such events but without evidence for mass clustering around the $60 \mathrm{Gev}$ level.

The conclusions are that one must continue with the analysis of many more events at the $Z$ energy as the number of collected events increases, and think more about possible sources of background. The situation is neatly summed up by the comment that "Ting has the event and others the background" - an event that according to a senior member of the L3 collaboration "is a good event - it has been attacked from all sides but it's still there". 